\title{
Regression of pyuria during the treatment of symptomatic urinary tract infection in patients with spinal cord injury
}

\author{
Anand Joshi and Rabih O Darouiche \\ Department of Physical Medicine and Rehabilitation, Spinal Cord Injury Service, and Department of Medicine, \\ Infectious Disease Section, Baylor College of Medicine and Veterans Affairs Medical Center, Houston, Texas, USA
}

\begin{abstract}
Pyuria is frequently present in patients who require bladder instrumentation. Using the hemocytometer chamber method, we prospectively studied the regression of pyuria in 29 spinal cord-injured (SCI) men with symptomatic urinary tract infection (UTI) who were grouped according to the method of bladder drainage: (a) Intermittent catheterization program (ICP; 10 patients); (b) Suprapubic tube (SPT; 10 patients); and (c) Indwelling foley catheters (IFC; 9 patients). All of the patients experienced relief of presenting. symptoms within 3-4 days of receiving appropriate antibiotic therapy. The clinical response was associated with $\geqslant 65 \%$ and $\geqslant 87 \%$ reduction in the levels of pyuria at mid-therapy and after completion of antimicrobial therapy, respectively. Using a one-way analysis of variance, the group of patients who underwent ICP had significantly lower residual levels of pyuria at midtherapy and after completion of therapy when compared to the other two groups $(P<0.05)$. The findings of relatively lower absolute levels of pyuria in the ICP group $v s$ the SPT and IFC group of patients suggest that the response of pyuria to appropriate therapy for symptomatic UTI can be assessed better and earlier in patients who undergo ICP.
\end{abstract}

Keywords: pyuria; urinary tract infection; spinal cord injury

\section{Introduction}

Pyuria is commonly associated with instrumentation of the bladder. In spinal cord injured (SCI) patients who require chronic bladder catheterization, the presence of white blood cells (WBC) in the urine may be caused by either a urinary tract infection (UTI) or by a variety of other conditions such as mechanical irritation, renal stones, renal tuberculosis, or extreme dehydration. ${ }^{1}$ The regression of urinary leukocyte response during treatment of symptomatic UTI has been previously studied using the centrifugation-reconstitution method. ${ }^{2}$ This method is conventionally used to quantify pyuria in most medical centers in the United States because it is inexpensive and technically simple. However, the centrifugation-reconsititution method suffers from poor reproducibility because of the potential variabilities in the size of the pellet (dependent on volume of centrifuged urine sample and duration and speed of centrifugation), volume of fluid used for reconstitution of the pellet, and the subjectivity of the reader in choosing microscopic fields for counting. The regression of pyuria during the treatment of symptomatic UTI has not been previously reported using a more accurate and reproducible method of quantitating pyuria in uncentrifuged urine. One such method is the microtitre tray and inverted microscope method which is more

Correspondence: RO Darouiche commonly used in European countries, particularly the United Kingdom. ${ }^{3}$ Commercial disposable standard volume slides are also available for use in these countries. Of all methods used for quantitating pyuria in uncentrifuged specimens of urine the hemocytometer chamber method is probably the most accurate, though relatively more laborious. ${ }^{4}$

This prospective study was conducted to (1) evaluate the regression of pyuria during treatment of UTI using the hemocytometer chamber method and (2) examine differences in regression trends of pyuria in SCI patients who require different modes of bladder catheterization.

\section{Methods}

\section{Subjects}

Male SCI patients who developed symptomatic UTI while hospitalized at the Veterans Affairs Medical Center, Houston, Texas, USA, were eligible for enrollment in this study. Symptomatic UTI was defined by the presence of significant pyuria $\left(>10^{7}\right.$ $\mathrm{WBC} / \mathrm{L})$ and bacteriuria $\left(\geqslant 10^{8} \mathrm{CFU} / \mathrm{L}\right)$ in association with fever $\left(\geqslant 38.6^{\circ} \mathrm{C}\right)$, leukocytosis, acute abdominal or suprapubic discomfort, change in voiding habits, autonomic dysreflexia, or worsening of muscle spasm for which no other etiology could be found. 
Enrolled patients were stratified to one of three groups according to the mode of bladder catheterization: (a) Intermittent catherization program (ICP); (b) Suprapubic tube (SPT); and (c) Indwelling foley catheter (IFC). A total of 41 eligible patients was consecutively enrolled with the objective of having a total of 10 evaluable patients in each group. Twelve of the 41 enrolled subjects were excluded from analysis either because of the presence of other possible etiologies for their symptoms, such as renal stones (six patients), pneumonia (three patients), deep vein thrombosis (one patient), or because at some point during the course of antibacterial treatment there was a documented superinfection of the urinary tract with Candida species that required antifungal therapy (two patients).

After obtaining initial urine samples for quantification of pyuria in uncentrifuged urine using the hemocytometer chamber method ${ }^{4}$ and for bacterial culture and antibiotic susceptibilities, patients were started on empiric antibiotic therapy (guided by previous findings from the most recent urine culture). If necessary, the initial antibiotic regimen was adjusted after obtaining results of pre-enrollment urine cultures; in these cases, the data obtained on the day the antibiotic therapy was adjusted represented initial values. A 7-day course of appropriate antibiotic therapy was given to all patients. Patients were clinically monitored every day until resolution of symptoms of UTI. Urine samples were obtained again at mid-therapy (day 3-4), and as well at the end of therapy (day 7-8) for measurement of pyuria and for culture.

\section{Results}

A total of 29 patients were evaluated (10 patients in the ICP group; 10 patients in the SPT group; and nine patients in the IFC group). The mean age of the 29 evaluable patients was 45 years, with a median of 38 years and a range of 28 to 61 years. All patients had complete neurologic lesions (ASIA 'A' classification) anywhere from $\mathrm{C} 5$ to T8. The duration of SCI varied from 68 days to several years. There were no differences between the three groups of patients as to age, gender, level of injury or duration of injury. Bladder management was based upon urodynamic evaluation, and a significant number $(41 \%)$ of patients had been receiving autonomic agents to modify vesicourethral action. Only three patients were receiving antimicrobial urinary prophylaxis (macrodantin or trimethoprim-sulfamethoxazole), but in each of these three cases the pathogenic organism proved to be resistent to the prophylactic antimicrobial agent.

At least one pathogenic organism was isolated from each initial urine culture, and multiple organisms were isolated from $18 / 29(62 \%)$ initial urine cultures. The most commonly isolated organism was Pseudomonas aeruginosa $(76 \%)$, followed by Klebsiella pneumoniae
$(46 \%)$, then Enterococcus sp $(36 \%)$. The empirically chosen antibiotic regimen provided coverage for at least one bacterial pathogen in 25/29 (86\%) cases and for all isolated organisms in $15 / 29(52 \%)$ cases, reflecting the ready applicability of results from previous urine cultures. The 14 cases in whom initially administered antibiotic therapy had to be adjusted were equally divided among the three groups of patients (five cases in each of the ICP and SPT groups and four cases in the IFC group). Moreover, there were no significant differences between these three groups as to the extent of reduction of pyuria levels from the time of onset of symptoms till adjustment of antibiotic therapy 2-3 days later. Symptoms attributable to UTI were noted to improve by day 1 of appropriate antibiotic therapy and were resolved by day 4 in all cases.

Pyuria progressively decreased during appropriate antibiotic treatment for UTI in SCI persons who require bladder instrumentation (Table 1). A planned contrast analysis was conducted with one-way repeat analysis of variance (ANOVA) within each group, demonstrating a substantial decline in pyuria values with time $(P<0.005)$ for each group. However, while the urinary WBC count normalized at the end of therapy for persons who underwent ICP (mean of $0.8 \times 10^{7} \mathrm{WBC} / \mathrm{L}$ ), this was not true for those who had SPT or IFC. In the latter two groups of patients, there were substantial residual levels of pyuria after completion of treatment (a mean of $5.8 \times 10^{7} \mathrm{WBC} / \mathrm{L}$ for the SPT group and a mean of $5.4 \times 10^{7} \mathrm{WBC} / \mathrm{L}$ for the IFC). Using a one-way ANOVA for the final evaluation (day 7-8), the residual levels of pyuria both at mid-therapy and after completion of therapy were significantly lower in the ICP group when compared to the other two groups $(P<0.05)$.

Table 1 Levels of pyuria in patients with different modes of bladder instrumentation

\begin{tabular}{lccc}
\hline $\begin{array}{l}\text { Bladder } \\
\text { instrumentation }\end{array}$ & $\begin{array}{c}\text { Level } \\
\text { Initial }\end{array}$ & $\begin{array}{c}\text { pyruia }\left(\mathrm{WBC} \times 10^{7} / \mathrm{L}\right) \\
\text { Mid-therapy }\end{array}$ & Final \\
\hline $\begin{array}{l}\text { ICP (10 patients) } \\
\text { Mean }\end{array}$ & 28.5 & 3.6 & 0.8 \\
Median & 26.0 & 6.0 & 1.0 \\
Range & $3.5-84.0$ & $0.0-11.5$ & $0.0-2.0$ \\
SD & 23.2 & 3.5 & 0.8 \\
SPT (10 patients) & & & \\
Mean & 43.9 & 15.3 & 5.8 \\
Median & 75.0 & 22.0 & 8.5 \\
Range & $6.0-161.0$ & $0.5-80$ & $0.5-21.0$ \\
SD & 46.1 & 23.6 & 6.1 \\
IFC (9 patients) & & & \\
Mean & 174 & 12.8 & 5.4 \\
Median & 175 & 12.0 & 6.0 \\
Range & $29-286$ & $3.5-35.5$ & $1.5-11.0$ \\
SD & 71.7 & 10.2 & 3.4 \\
\hline
\end{tabular}

ICP $=$ Intermittent catheterization program; SPT $=$ Suprapubic tube; IFC = Indwelling Foley catherter) 


\section{Discussion}

The findings of this study demonstrated that in SCI patients who are being treated for symptomatic UTI, the resolution of clinical symptoms is associated with a progressive decrease in the level of pyuria. This seems to be generally true in SCI patients, irrespective of the mode of bladder catheterization. For all three groups of patients enrolled in this study, the largest decrease in leukocyte response occurred during the first 3-4 days of treatment (ie from the beginning to mid-therapy), averaging $87 \%$ for the ICP group, $65 \%$ for the SPT group and $93 \%$ for the IFC group. In all cases, the large decrease in leukocyte response during this initial period was associated with a complete resolution of symptoms attributable to UTI. The absolute decline in level of pyuria from mid-therapy to completion of treatment was small when compared to the drop during the first half of therapy course. At the end of antibiotic therapy, the mean WBC count in urine had dropped by at least $87 \%$ of its initial value in all of the three groups of patients. Such a decline in the levels of pyuria is in agreement with previously reported results in ICP-requiring patients who develop UTI due to gram-negative bacteria. ${ }^{3}$ The findings of relatively lower absolute levels of pyuria in the ICP group vs the SPT and IFC group of patients in this study suggest that the response of pyuria to therapy for symptomatic UTI can be assessed better and earlier in patients who undergo ICP.
The demonstration of improvement of symptoms in association with a decline of pyuria during the first 3-4 days of appropriate treatment for symptomatic UTI may have important clinical implications. In SCI patients who have significant bacteriuria and pyuria in association with non-specific clinical manifestations (such as fever, autonomic dysreflexia and worsening spasms), it is often unclear whether these patients truly have a clinical UTI or just have asymptomatic UTI in association with pressure ulcer, heterotopic ossification, or another condition which might cause such nonspecific manifestations. In such patients who receive seemingly appropriate antibiotic therapy for presumed clinical UTI, the persistence of these non-specific clinical manifestations despite the regression of pyuria and bacteriuria may encourage the search for other conditions that may co-exist with asymptomatic UTI.

\section{References}

1 O'Riley PH, Edwards EC, Cope V. Abacterial pyuria. Brit J Radiol 1980; 53: 494-495.

2 Lewis DA. Bacteriology of Urine. Hawkey PM and Lewis DA (eds) Medical Bacteriology: A Practical Approach. IRL Press and Oxford University Press: Oxford, UK, 1989.

3 Anderson RU, Hsieh-Ma ST. Association of bacteriuria and pyuria during intermittent catheterization after spinal cord injury. J Urol 1983; 130: $299-301$.

4 Musher DM, Thorsteinsson SB, Airola VM. Quantitative urinalysis: diagnosing urinary tract infection in men. $J$ Amer Med Assoc 1976; 236: 2069-2072. 